\title{
Code Design for Binary Energy Harvesting Channel
}

\author{
Mehdi Dabirnia and Tolga M. Duman \\ Dept. of Electrical and Electronics Engineering, Bilkent University, Bilkent 06800, Ankara, Turkey \\ Email: \{mehdi, duman\}@ee.bilkent.edu.tr
}

\begin{abstract}
We consider a binary energy harvesting communication system with a finite battery transmitter over a noisy channel, and design explicit and implementable codes based on concatenation of a nonlinear trellis code (NLTC) with an outer low density parity check (LDPC) code. We propose two different decoding methods where the simplified one ignores the memory in the battery state while the more sophisticated one utilizes the memory. Numerical results demonstrate that the designed codes outperform other reference schemes. The results also show the superiority of the improved decoding approach over the naive solution.
\end{abstract}

Keywords-Binary energy harvesting channel, nonlinear trellis codes, LDPC code design, channels with memory.

\section{INTRODUCTION}

We consider a single user communication system in which the transmitter derives the required energy for information transmission from an external source. The transmitter transmits a symbol through the channel with respect to the availability of energy in the battery. It also harvests energy and stores it in a battery for subsequent transmissions. Energy harvesting communication systems have been studied recently from an information theoretic point of view in the literature. It has been shown in [1] that for the case of infinite battery, the capacity of an additive white Gaussian noise (AWGN) channel with the average power constraint equal to the average recharge rate can be achieved. The case with no battery over an AWGN channel has been studied in [2], and an analysis of the channel capacity has been provided. Finite size battery case has been studied in [3]-[5], and approximations along with bounds on the capacity have been obtained, however, the capacity for this case is still open. Authors in [6], [7] consider binary energy harvesting noiseless channels with unit-sized battery, derive a channel capacity formula by utilizing an equivalent timing channel, and provide computable upper and lower bounds.

While many advancements have been made from an information theoretic perspective, explicit code design for the binary energy harvesting noisy channel has not been studied previously. With this motivation, we consider this model with finite battery and design explicit and implementable codes based on a serially concatenated coding scheme. We consider using independent and identically distributed (i.i.d.) on-off signaling and derive the optimal ones' density for different energy arrival probabilities and battery capacities. Observing that the optimal input distribution is nonuniform we propose an encoding scheme based on concatenation of an inner nonlinear trellis code (NLTC) with an outer low density parity check (LDPC) code. The inner nonlinear code is used to provide the optimal ones' density while the outer linear code offers strong

This work was supported by the European Commission Marie Curie Career Integration under Grant PCIG12-GA-2012-334213. error correction capabilities. We note that, other approaches, e.g., based on "probabilistic shaping" [8] or "constrained coding" [9] can be employed for this problem. At the receiver side, as a simple decoding approach, we ignore the memory in the battery state and consider a channel with i.i.d states. We calculate the stationary probability of the zero battery state $\left(\pi_{0}\right)$ and consider a memoryless $\mathrm{Z}$ channel with $1 \rightarrow 0$ crossover probability $\pi_{0}$ connected to the corresponding channel model. The receiver performs iterative decoding over the equivalent memoryless channel. Due to its simplicity, this decoding scheme is utilized for code design purposes, i.e., for finding the optimal LDPC code ensembles. The scheme obtained by this simple strategy is called the naive i.i.d. Shannon strategy (NIID) and the corresponding achievable rates are obtained in [6]. This scheme is clearly sub-optimal because it ignores the memory in the system.

Another main contribution of this study is the development of an improved alternative decoding scheme for the system model under consideration. In order to take advantage of the memory in the battery state at the decoder side, we build an extended trellis representing both the NLTC and battery states, and perform BCJR decoding on this extended trellis. The scheme obtained by this modified decoding strategy is called optimal i.i.d. Shannon strategy (OIID) and the corresponding achievable rates are obtained in [3], [6]. Through several numerical examples, we demonstrate that the proposed decoder is highly superior to the simple decoding approach.

The paper is organized as follows. In Section II, we introduce the system model for a binary energy harvesting communication system, and provide achievable rates and optimal ones' densities for different channel models. Proposed encoding and decoding schemes are presented in Section III along with the code design procedure. We provide several code design examples and decoding performance results in Section IV, and finally, we conclude the paper in Section V.

\section{SYSTEM DESCRIPTION}

We consider the model introduced in [6] for an energy harvesting channel in which the energy arrivals $\left(E_{i}\right)$ are binary (i.e., either zero or one unit of energy arrives, $E_{i} \in\{0,1\}$ ). The channel inputs are binary as well, i.e., $X_{i} \in\{0,1\}$ (with zero or one unit energy cost). Battery size is a multiple of the fixed unit of energy harvests (denoted by $B_{\max }$ ). We assume an i.i.d. energy arrival process $\left(E_{i} \sim \operatorname{Bernoulli}(q)\right)$, however, the specific coding scheme proposed in Section III can be extended easily to other energy arrival models such as those incorporating Markov arrivals as well.

By observing the battery state $S_{i}$, for each channel use, the transmitter first transmits a symbol $X_{i}$, and then it harvests energy $E_{i}$ and stores it in the battery if there is space. If the 
battery is empty, regardless of what the input bit is, $X_{i}=0$ is transmitted. The battery state evolves as $S_{i+1}=\min \left\{S_{i}-\right.$ $\left.X_{i}+E_{i}, B_{\max }\right\}$, and it is causally known at the transmitter only. In this model, we have a state dependent channel for which the channel state $S_{i}$ depends on the channel input $X_{i}$, hence it has memory. Since the channel state is not i.i.d. over time, i.i.d. Shannon strategy does not give the capacity for this channel, however, it can provide an achievable rate [3].

We compute achievable rates based on the i.i.d. Shannon strategies for binary symmetric channel (BSC) and AWGN channel for illustration. We consider the encoding scheme with i.i.d. on-off signaling and ones' density $\left(P\left(X_{i}=1\right)=p\right)$. Since energy arrivals are i.i.d., the battery state process can be modelled as a Markov chain shown in Fig. 1. The stationary probabilities of the battery states are given as

$$
\begin{gathered}
P(S=0)=\pi_{0}=\frac{(1-q) p}{(1-q) p+q \sum_{i=0}^{B \max -1}(q / p)^{i}} \\
P(S=i)=\pi_{i}=\frac{1}{1-q}\left(\frac{q}{p}\right)^{i} \pi_{0}, i=1, \ldots, B_{\max } .
\end{gathered}
$$
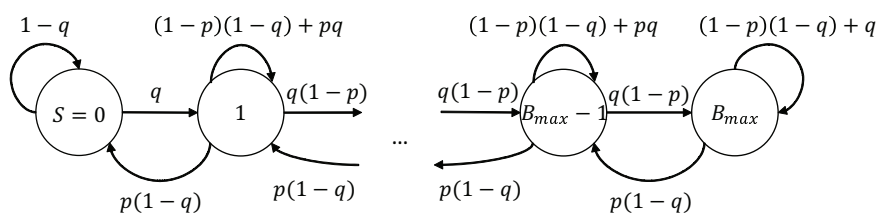

Fig. 1: Markov chain model of the battery state with battery capacity $B_{\max }$.

If the receiver ignores the memory in the model and considers a channel with i.i.d. states with the state probabilities given above, namely, using NIID [6], the achievable rate over a BSC with crossover probability $\epsilon$ and an AWGN channel with noise variance $\frac{N_{0}}{2}$ are given as

$R_{N I I D-B S C}=\max _{p \in[0,1]} H_{2}\left(p\left(\left(1-\pi_{0}\right)(1-\epsilon)+\pi_{0} \epsilon\right)+(1-p) \epsilon\right)$ $-p H_{2}\left(\left(1-\pi_{0}\right)(1-\epsilon)+\pi_{0} \epsilon\right)-(1-p) H_{2}(\epsilon)$,

$R_{N I I D-A W G N}=\max _{p \in[0,1]} I(Y ; M)=\max _{p \in[0,1]} h(Y)-h(Y \mid M)$,

respectively, where $H_{2}($.$) is the binary entropy function, and$ $Y \sim\left((1-p)+p \pi_{0}\right) \mathcal{N}\left(0, N_{0} / 2\right)+p\left(1-\pi_{0}\right) \mathcal{N}\left(1, N_{0} / 2\right)$ $Y \mid M \sim \begin{cases}\mathcal{N}\left(0, N_{0} / 2\right) & \text { if } M=0, \\ \pi_{0} \mathcal{N}\left(0, N_{0} / 2\right)+\left(1-\pi_{0}\right) \mathcal{N}\left(1, N_{0} / 2\right) & \text { if } M=1,\end{cases}$

and $\mathcal{N}\left(0, N_{0} / 2\right)$ represents the zero mean Gaussian distribution with variance $\frac{N_{0}}{2}$. Note that in both (3) and (4) the parameter $\pi_{0}$ depends on the ones' density $p$. For the AWGN case, the signal to noise ratio is defined as the ratio of energy arrival rate to the noise power, namely, $\mathrm{SNR}=\frac{q}{N_{0}}$.

The optimal ones' density $p$ to achieve $R_{N I I D-B S C}$ over a $\operatorname{BSC}(0.1)$ is depicted in Fig. 2. Clearly, the optimal ones' density in most cases is not uniform which suggests that using linear block codes which induce a uniform input distribution of " 0 "s and " 1 "s, by themselves is not optimal, namely, one

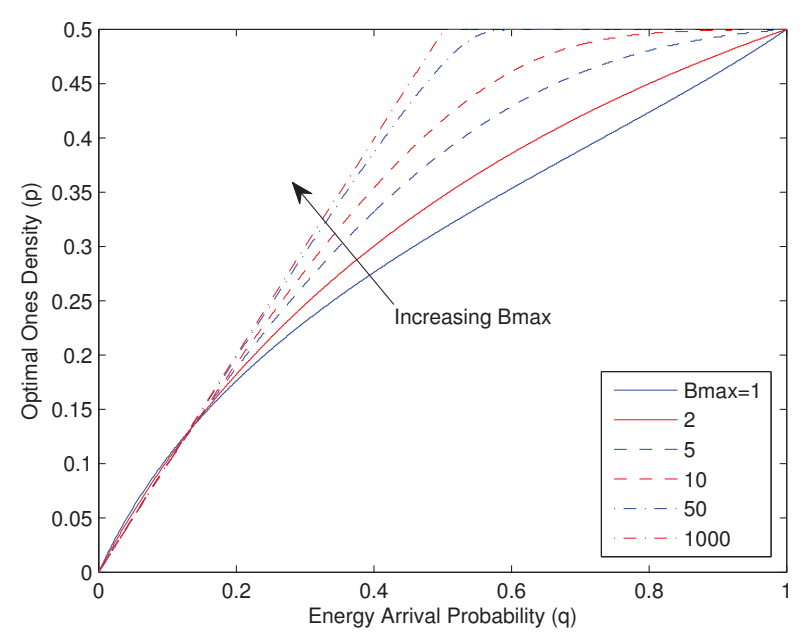

Fig. 2: Optimal ones' density for the NIID scheme for binary input energy harvesting $\operatorname{BSC}(0.1)$.

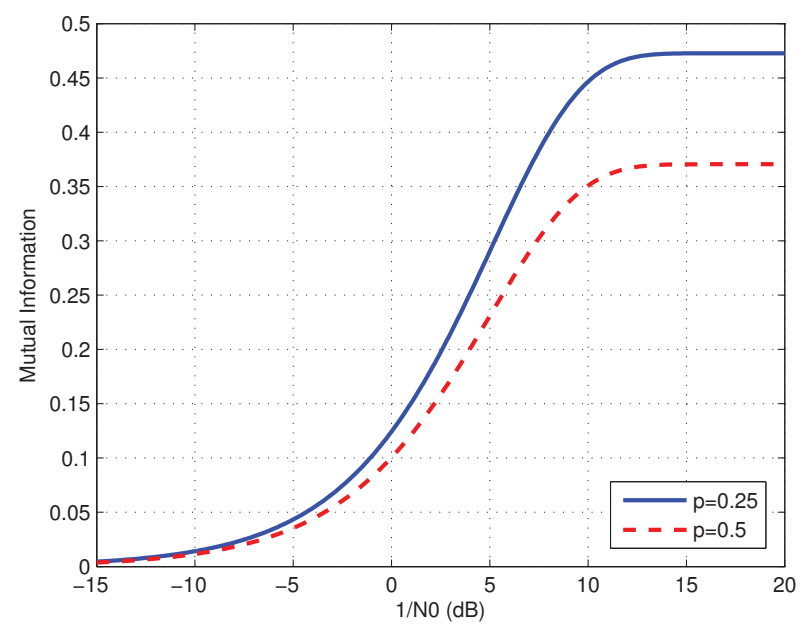

Fig. 3: Achievable rates with the NIID scheme over an AWGN channel with $q=0.4$.

can obtain higher rates by using nonlinear codes for reliable information transmission.

When the battery capacity increases, the optimal ones' density approaches the energy arrival probability for the interval $0<q<0.5$, and it becomes uniform for $0.5 \leq q \leq 1$. Another interesting observation from Fig. 2 is that when the battery capacity is small and energy arrival probability is also very small, e.g., $q=0.12$ or lower, the optimal ones' density is larger than the energy arrival probability.

Fig. 3 depicts the achievable rates for the special case of $q=0.4$ over an AWGN channel for which the ones' density $p=0.25$ is optimal for high SNRs. As it is shown in this figure, by using a ones' density of $p=0.25$, higher rates compared to those of uniform inputs can be achieved.

\section{PROPOSED CODING SCHEME}

As described in the previous section, in order to achieve $R_{N I I D}$, a specific input distribution at the channel input (which is usually nonuniform) needs to be employed. To generate the required nonuniform input distribution and also 


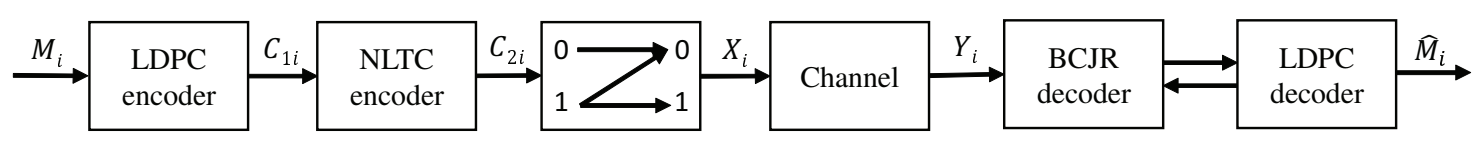

Fig. 4: Block diagram of the proposed coding scheme.

to obtain good error correction performance, in this paper, we propose concatenation of an outer linear block code such as an LDPC code with an inner nonlinear trellis code as a practical approach.

Fig. 4 depicts the block diagram of the proposed coding scheme. The transmitter side consists of concatenation of an outer LDPC encoder with an inner nonlinear trellis encoder with a battery state conditioner, which is then connected to the channel. Battery state conditioner simply applies the battery outage effect which is nothing but transmitting symbol 0 when the battery is empty. Considering an LDPC code of rate $R_{1}$ and an NLTC of rate $R_{2}$, the overall code rate is equal to $R_{1} R_{2}$. The goal of the inner NLTC is to induce the desired nonuniform distribution at the channel input while providing error protection against channel noise. The outer LDPC code is intended to provide error correction capabilities.

At the receiver side, as a simple approach, we first ignore the memory in the channel state and consider a channel with i.i.d. states with stationary probabilities given in (1)-(2). As a result we have a memoryless $\mathrm{Z}$ channel with crossover $1 \rightarrow 0$ probability $\pi_{0}$ connected to the memoryless noisy channel (which is either a BSC or an AWGN channel in our setting). Then a BCJR algorithm based decoder is adopted for the two step memoryless channel computing the log-likelihood-ratios (LLRs) of the encoded bits to be fed to the LDPC decoder. To improve the decoding performance, the soft outputs of the LDPC decoder are fed back to the BCJR decoder in an iterative fashion as also illustrated in Fig. 4. The decoding scheme described above is used for the code design purposes in the next section, however, we introduce another (improved) iterative decoding solution in Section III-C which exploits the memory in the battery state as well.

\section{A. Inner NLTC Design}

To design the inner NLTC, we utilize the algorithm developed in [10] which maximizes the minimum distance of the code while keeping a desired ones' density. As it is stated in [11], having a larger minimum distance helps with the stability of the iterative decoding process. Also, it is a valid criterion for designing trellis-base codes for point-to-point (P2P) scenarios. The output of the design algorithm is a lookup table which assigns specific labels to the branches of the trellis in such a way that ensures the specific distribution of ones and zeros, and provides a large minimum distance.

\section{B. Outer LDPC Code Design}

Off-the-shelf LDPC codes that are optimized for $\mathrm{P} 2 \mathrm{P}$ communication scenarios (e.g., for AWGN channels) are not optimal for the case of energy harvesting communication systems, that is, one needs to optimize the degree distribution of the LDPC ensemble (for each specific energy harvesting scenario). To accomplish this, we fix the inner NLTC (which is specifically designed to provide the optimal ones' density for the particular energy harvester parameters, e.g., battery size, energy arrival probability), and perform optimization of the LDPC degree distribution. LDPC code optimization can be done based on different objectives such as decoding threshold optimization or rate maximization. In this paper, we employ the latter approach. Among different algorithms and techniques available, we utilize the EXIT analysis [12] to measure the iterative decoding threshold of the concatenated coding scheme, and employ the random perturbation algorithm to generate new instances of the LDPC degree distribution.

We assume symmetry of the PDF of the exchanged LLRs between the component decoders, and perform the EXIT analysis by using Monte Carlo simulations without any assumption on the Gaussianity of the exchanged LLRs [11]. We follow the approach taken in [13] and compute the extrinsic mutual information using

$$
I(L ; X) \approx 1-\frac{1}{N} \sum_{n=1}^{N} \log _{2}\left(1+e^{-L_{n}}\right)
$$

with $L_{n}$ denoting the LLR corresponding to the $n$th coded bit of the all-zero codeword. We utilize i.i.d. channel adaptors introduced in [14] with common randomness at the transmitter and the receiver sides for each message to validate the allzero codeword transmission assumption. Using Monte Carlo simulations for sufficiently long block lengths (taken as $10^{6}$ in this study) for the iterative decoder, and tracking the evolution of the mutual information at the output of the component decoders, we check for convergence. We use perturbing vectors to generate new instances of the degree distributions with the goal of maximizing the rate of the code. Then, we check the convergence of the iterative decoding for the new degree distribution ensemble using the EXIT analysis. We terminate the optimization procedure if no improvement can be obtained after a predetermined number of iterations. Further details about the LDPC code design by using random perturbation technique can be found in [11], [15].

\section{An Improved Iterative Decoding Algorithm}

As a second decoding approach, we propose building an extended trellis by including the battery state at the beginning of each trellis section along with the NLTC states which expands the size of the trellis by a factor of $B_{\max }+1$, i.e., for each NLTC state, there are $B_{\max }+1$ corresponding states in the extended trellis, namely, one for each battery state in $\left\{0,1, \ldots, B_{\max }\right\}$. Each transition in the NLTC trellis corresponds to $\left(B_{\max }+1\right)^{2}$ transitions in the extended trellis, however, some of these transitions might be invalid depending on the current and the next battery states and the output label in the NLTC.

Let us illustrate this idea by a simple example. Consider a simple two state NLTC given in Fig. 5. Assuming that the energy harvester is equipped with a unit-sized battery, a section of the extended trellis diagram for this code is shown in Fig. 6. The state $S_{i} / x$ corresponds to the case where the NLTC state is $S_{i}$ and the battery state is $x$. The 
labels corresponding to each transition of the extended trellis along with their probabilities are given in Table I where $\bar{q}=1-q$. In this approach, the BCJR decoder operates on the extended trellis and exchanges the soft extrinsic LLRs with the LDPC decoder in an iterative manner. Complexity increase for the improved decoder compared to the simple decoding scheme is at most quadratic which can be tolerated even for code design purposes, however, we do not use this scheme for designing codes in this paper. We demonstrate the superior performance of this approach via finite block length bit error rate simulations using the specific codes taken from the designed ensembles of the previous subsection. We also note that, reduced complexity trellis-based decoders can also be employed to obtain computationally simpler decoding solutions while still utilizing the extended trellis.

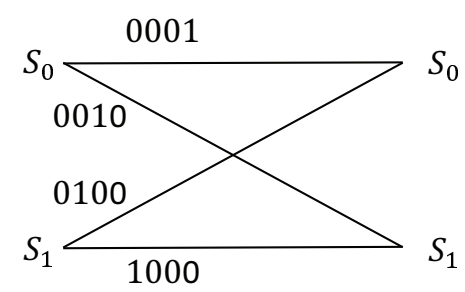

Fig. 5: The 2-state NLTC.

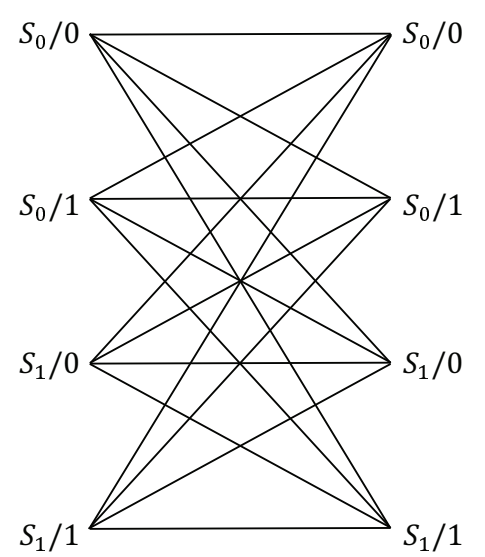

Fig. 6: The extended trellis section for 2-state NLTC and unitsized battery.

\section{NUMERICAL EXAMPLES}

As a first example, we consider an energy harvester with a unit-sized battery and energy arrival probability of $q=0.4$ over an AWGN channel for which the achievable rates using ones' density of 0.25 and 0.5 are shown in Fig. 3. We consider a ones' density $p=0.25$ which is the optimal ones' density for this energy arrival probability at high SNRs and design an NLTC satisfying this constraint while maximizing the minimum distance of the code. Details of the designed trellis code are given in Table II. In the next step, by fixing the inner NLTC, the outer LDPC code is designed by maximizing the rate of the code for a convergence threshold of $0 \mathrm{~dB}$ for which the result is given in Table III. Also concatenation of a nonlinear memoryless mapper (NLMM) (of the same rate and ones' density as the designed NLTC) with an optimized outer LDPC code is considered as reference.
TABLE I: Labels corresponding to the transitions of the extended trellis and their probabilities.

\begin{tabular}{|c|c|c|c|}
\hline Current State & Next State & Output Label & Probability \\
\hline \hline \multirow{2}{*}{$S_{0} / 0$} & \multirow{2}{*}{$S_{0} / 0$} & 0000 & $\bar{q}^{4}$ \\
\cline { 3 - 4 } & & 0001 & $\left(1-\bar{q}^{3}\right) \bar{q}$ \\
\hline \multirow{2}{*}{$S_{0} / 0$} & \multirow{2}{*}{$S_{0} / 1$} & 0000 & $\bar{q}^{3} q$ \\
\cline { 3 - 4 } & & 0001 & $\left(1-\bar{q}^{3}\right) q$ \\
\hline$S_{0} / 1$ & $S_{0} / 0$ & 0001 & $\bar{q}$ \\
\hline$S_{0} / 1$ & $S_{0} / 1$ & 0001 & $q$ \\
\hline \multirow{2}{*}{$S_{0} / 0$} & \multirow{2}{*}{$S_{1} / 0$} & 0000 & $\bar{q}^{4}$ \\
\cline { 3 - 4 } & & 0010 & $\left(1-\bar{q}^{2}\right) \bar{q}^{2}$ \\
\hline \multirow{2}{*}{$S_{0} / 0$} & $S_{1} / 1$ & 0000 & $\bar{q}^{2}\left(1-\bar{q}^{2}\right)$ \\
\cline { 3 - 4 } & & 0010 & $\left(1-\bar{q}^{2}\right)^{2}$ \\
\hline$S_{0} / 1$ & $S_{1} / 0$ & 0010 & $\bar{q}^{2}$ \\
\hline$S_{0} / 1$ & $S_{1} / 1$ & 0010 & $1-\bar{q}^{2}$ \\
\hline \multirow{2}{*}{$S_{1} / 0$} & \multirow{2}{*}{$S_{0} / 0$} & 0000 & $\bar{q}^{4}$ \\
\cline { 3 - 4 } & & 0100 & $q \bar{q}^{3}$ \\
\hline \multirow{2}{*}{$S_{1} / 0$} & \multirow{2}{*}{$S_{0} / 1$} & 0000 & $\bar{q}\left(1-\bar{q}^{3}\right)$ \\
\cline { 3 - 4 } & & 0100 & $q\left(1-\bar{q}^{3}\right)$ \\
\hline$S_{1} / 1$ & $S_{0} / 0$ & 0100 & $\bar{q}^{3}$ \\
\hline$S_{1} / 1$ & $S_{0} / 1$ & 0100 & $1-\bar{q}^{3}$ \\
\hline$S_{1} / 0$ & $S_{1} / 0$ & 0000 & $\bar{q}^{4}$ \\
\hline$S_{1} / 0$ & $S_{1} / 1$ & 0000 & $1-\bar{q}^{4}$ \\
\hline$S_{1} / 1$ & $S_{1} / 0$ & 1000 & $\bar{q}^{4}$ \\
\hline$S_{1} / 1$ & $S_{1} / 1$ & 1000 & $1-\bar{q}^{4}$ \\
\hline
\end{tabular}

TABLE II: Label assignment to the branches of 16-state trellis $(M=4)$ for the designed NLTC.

\begin{tabular}{|c|c|c|c|c|c|c|c||c|}
\hline \multicolumn{7}{|c|}{ State/Input } & Output \\
\hline \hline $0000 / 0$ & $0001 / 1$ & $0100 / 1$ & $0101 / 0$ & $1010 / 0$ & $1011 / 1$ & $1110 / 1$ & $1111 / 0$ & 0001 \\
\hline $0000 / 1$ & $0001 / 0$ & $0100 / 0$ & $0101 / 1$ & $1010 / 1$ & $1011 / 0$ & $1110 / 0$ & $1111 / 1$ & 0010 \\
\hline $0010 / 0$ & $0011 / 1$ & $0110 / 1$ & $0111 / 0$ & $1000 / 0$ & $1001 / 1$ & $1100 / 1$ & $1101 / 0$ & 0100 \\
\hline $0010 / 1$ & $0011 / 0$ & $0110 / 0$ & $0111 / 1$ & $1000 / 1$ & $1001 / 0$ & $1100 / 0$ & $1101 / 1$ & 1000 \\
\hline
\end{tabular}

To study the performance of the specific codes from the designed ensemble, parity check matrices for a block-length of $10 \mathrm{k}$ are obtained using the tools in [16] where the length-4 cycles are removed for improved performance. The resulting bit error rates are depicted in Fig. 7. The overall code rate for both schemes are 0.126 . We observe that the designed codes via the proposed coding solution outperform the reference scheme by about $2.5 \mathrm{~dB}$ at a bit error rate of $10^{-3}$. Furthermore the superior performance of the improved decoding scheme compared to the simple one (by about $0.25 \mathrm{~dB}$ at the same bit error rate value) can be observed from the simulations.

TABLE III: Optimized degree distribution of the outer LDPC code for the proposed coding scheme.

\begin{tabular}{|c||c|c|c|c|c|c|}
\hline & $\lambda_{2}$ & $\lambda_{3}$ & $\lambda_{4}$ & $\lambda_{5}$ & & \\
\hline Ex.1 (AWGN) & 0.492561 & 0.106999 & 0.399260 & 0.001180 & & \\
\hline Ex.2 (BSC) & 0.568342 & 0.067598 & 0.351750 & 0.012310 & & \\
\hline & $\rho_{2}$ & $\rho_{3}$ & $\rho_{4}$ & $\rho_{8}$ & $\rho_{1} 4$ & $\rho_{1} 5$ \\
\hline Ex.1 (AWGN) & 0.012700 & 0.405182 & 0.034768 & 0.027342 & 0.025157 & 0.494851 \\
\hline Ex.2 (BSC) & 0.000208 & 0.088826 & 0.004891 & 0.067288 & 0.254800 & 0.583986 \\
\hline
\end{tabular}

As a second example, we consider an energy harvester with a unit-sized battery and an energy arrival probability of $q=0.34$ over a BSC with crossover probability $\epsilon=0.1$. The optimal ones' density for this setup is $p=0.25$, hence we use the same NLTC as the one in the previous example. We optimize the outer LDPC code for this channel by fixing the inner NLTC and maximizing the rate of the LDPC code ensemble (see Table III for the resulting degree distributions). The achieved rates by using the proposed scheme and reference scheme are 0.1892 and 0.1741 , respectively. We note that the achieved rate of 0.1892 has a very small gap (of only 0.009) with the achievable information theoretic limit by using the NIID strategy. 


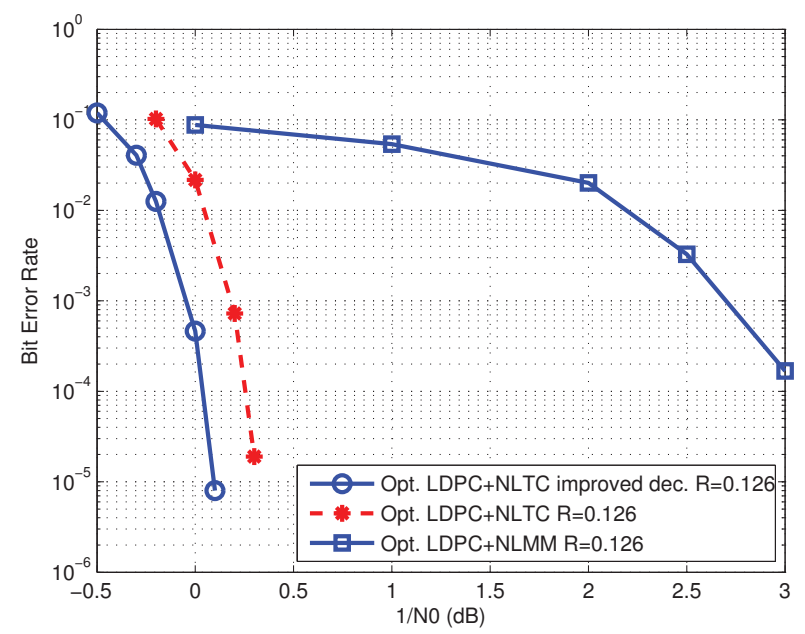

Fig. 7: Bit error rate performance of the proposed concatenated coding scheme with simple and improved decoding compared to that of the reference scheme $(q=0.4)$.

We also construct specific LDPC codes with block lengths of $10 \mathrm{k}$ using the optimized degree distributions employing the tools in [16], and perform bit error rate simulations. The results are illustrated in Fig. 8. It can be observed that the newly designed codes provide clear rate and error rate benefits compared to the reference scheme of using nonlinear memoryless mappers with optimized outer LDPC codes, and P2P optimal codes. In addition, simulation results show that, for example, at a crossover probability of $\epsilon=0.095$, an improvement of about two orders of magnitude in the bit error rate can be obtained by using the improved iterative decoding solution compared to the simple approach in Section III. Note that since the capacity is unknown for these examples, we do not provide a comparison with the information theoretic limits.

\section{CONCLusion}

In this paper, we have considered a binary energy harvesting communication system over noisy channels, and designed explicit and implementable codes based on a concatenated coding solution consisting of an inner NLTC and an outer LDPC code. At the receiver side, two decoding approaches are studied. The first one ignores the memory in the battery state, while the second one incorporates this memory into the trellis resulting in an extended trellis. For both cases, the BCJR decoder iteratively exchanges soft information with the LDPC decoder. To design the outer LDPC code, we utilize the first decoding scheme which is simpler. Through several numerical examples for different channel models, we illustrate that the newly designed codes outperform the alternatives of using P2P optimal codes and the reference scheme of utilizing nonlinear memoryless mappers with specifically optimized LDPC codes.

\section{REFERENCES}

[1] O. Ozel and S. Ulukus, "Achieving AWGN capacity under stochastic energy harvesting," IEEE Trans. Inf. Theory, vol. 58, no. 10, pp. 64716483, Oct. 2012.

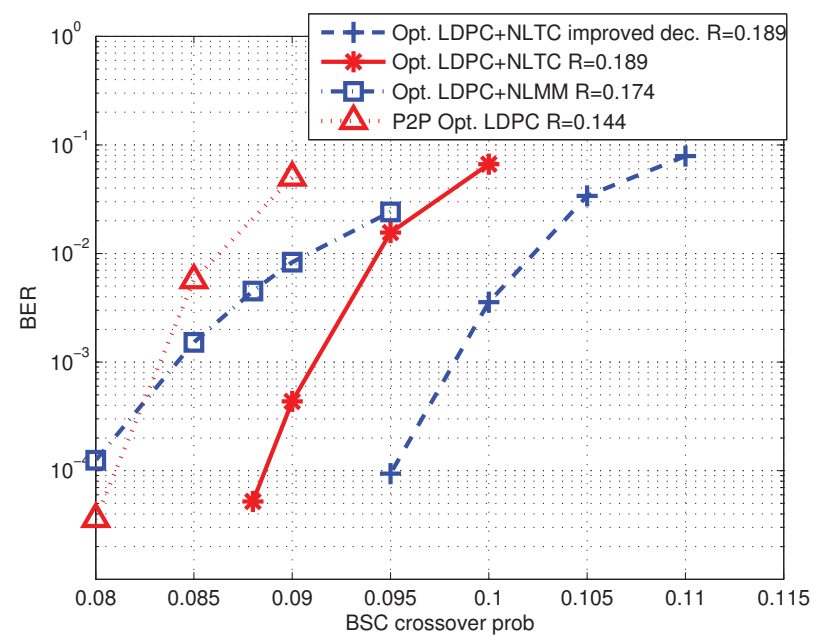

Fig. 8: Bit error rate performance of the proposed concatenated coding scheme and reference scheme $(q=0.34)$.

[2] O. Ozel and S. Ulukus, "AWGN channel under time-varying amplitude constraints with causal information at the transmitter," in Conf. Record of the Forty Fifth Asilomar Conference on Signals, Systems and Computers (ASILOMAR), Pacific Grove, CA, Nov. 2011, pp. 373377.

[3] W. Mao and B. Hassibi, "On the capacity of a communication system with energy harvesting and a limited battery," in Proc. IEEE Int. Symp. Information Theory (ISIT), Istanbul, Jul. 2013, pp. 1789-1793.

[4] Y. Dong and A. Ozgur, "Approximate capacity of energy harvesting communication with finite battery," in Proc. IEEE Int. Symp. Information Theory (ISIT), Honolulu, HI, Jun. 2014, pp. 801-805.

[5] V. Jog and V. Anantharam, "An energy harvesting AWGN channel with a finite battery," in Proc. IEEE Int. Symp. Information Theory (ISIT), Honolulu, HI, Jun. 2014, pp. 806-810.

[6] K. Tutuncuoglu, O. Ozel, A. Yener and S. Ulukus, "Binary energy harvesting channel with finite energy storage," in Proc. IEEE Int. Symp. Information Theory (ISIT), Istanbul, Jul. 2013, pp. 1591-1595.

[7] K. Tutuncuoglu, O. Ozel, A. Yener and S. Ulukus, "Improved capacity bounds for the binary energy harvesting channel," in Proc. IEEE Int. Symp. Information Theory (ISIT), Honolulu, HI, Jun. 2014, pp. 976-980.

[8] G. Bcherer and R. Mathar, "Operating LDPC codes with zero shaping gap," in Proc. IEEE Information Theory Workshop, Paraty, 2011, pp. 330-334.

[9] A. M. Fouladgar, O. Simeone, E. Erkip, "Constrained Codes for Joint Energy and Information Transfer", IEEE Trans. Commun., vol. 62, no. 6, pp. 2121-2131, Jun. 2014.

[10] M. Dabirnia and T. M. Duman, "On the code design for joint energy and information transfer," IEEE Trans. Commun., vol. 64, no. 6, pp. 2677-2688, Jun. 2016.

[11] M. Dabirnia, A. K. Tanc, S. Sharifi, and T. M. Duman, "Code design for discrete memoryless interference channel," in preparation.

[12] S. Ten Brink, "Convergence behavior of iteratively decoded parallel concatenated codes," IEEE Trans. Commun., vol. 49, no. 10, pp. 17271737, Oct. 2001.

[13] J. Hagenauer, "The EXIT chart - introduction to extrinsic information transfer in iterative processing," in Proc. 12th European Signal Processing Conference, Vienna, Sept. 2004, pp. 1541-1548.

[14] J. Hou, P. Siegel, L. Milstein, and H. Pfister, "Capacity-approaching bandwidth-efficient coded modulation schemes based on low-density parity-check codes," IEEE Trans. Inf. Theory, vol. 49, no. 9, pp. 21412155, Sep. 2003.

[15] S. Sharifi, A. K. Tanc, and T. M. Duman, "Implementing the HanKobayashi scheme using low density parity check codes over Gaussian interference channels," IEEE Trans. Commun., vol. 63, no. 2, pp. 337350, Feb. 2015.

[16] [Online]. Available: http://itpp.sourceforge.net/4.3.1/ 